\title{
Tuning thermal conductance across sintered silicon interface by local nanostructures
}

\author{
Masanori Sakata, ${ }^{\dagger}$ Takuma Hori, ${ }^{\dagger}$ Takafumi Oyake, ${ }^{\dagger}$ Jeremie Maire, ${ }^{t}$ Masahiro \\ Nomura, ${ }^{t}$ Junichiro Shiomi ${ }^{\dagger}, \xi$ \\ $†$ Department of Mechanical Engineering, The University of Tokyo, Tokyo 113-8656, \\ Japan \\ $\$$ Institute of Industrial Science, The University of Tokyo, Tokyo 153-8505, Japan \\ $\S$ PRESTO, Japan Science and Technology Agency, 4-1-8, Kawaguchi, Saitama, \\ 332-0012, Japan
}

Corresponding Author: shiomi@ photon.t.u-tokyo.ac.jp

\begin{abstract}
A large reduction of heat conduction through silicon-silicon sintered interface by local oxide nanostructures is quantitatively demonstrated by a newly developed method to directly measure thermal boundary conductance across bonded interfaces. Together with the theoretical analysis that relates the thermal boundary conductance to thermal conductivity of densely-packed bulk nanocrystalline silicon, we identify a route to significantly reduce the thermal conductivity from the state-of-art value, even to approach the amorphous silicon value. The finding is useful for designing nanostructured bulk silicon thermoelectrics
\end{abstract}

Keywords: Thermal boundary conductance, silicon, sintering, themoelectrics 


\section{Introduction}

Thermoelectric materials, which can convert thermal energy directly to electricity, have attracted great interest as a mean for clean, silent, long-life, and ubiquitous waste-heat recovery. The performance of a thermoelectric material can be quantified by the dimensionless figure-of-merit $Z T=S^{2} \sigma T /\left(k_{\mathrm{e}}+k_{\mathrm{ph}}\right)$, where $S$ is Seebeck coefficient, $\sigma$ is electrical conductivity, $k_{\mathrm{e}}$ and $k_{\mathrm{ph}}$ are thermal conductivity by electrons and phonons, and $T$ is temperature. A successful strategy to enhance $Z T$ has been to reduce lattice thermal conductivity without appreciably sacrificing the power factor $S^{2} \sigma$. For this, artificial nanostructuring has been particularly effective to inhibit heat conduction by scattering phonons at nanostructure-boundaries. Early studies on periodic nanostructures such as $\mathrm{Bi}_{2} \mathrm{Te}_{3} / \mathrm{Sb}_{2} \mathrm{Te}_{3}$ superlattice[1] and $\mathrm{PbSeTe}$ based quantum dot superlattice[2] marked high $Z T$, however, they have shortcoming in scalability and cost efficiency for practical applications. Recently, nanocrystalline materials (or nanostructured bulk composite), formed by compaction of nanopowder by sintering, have been demonstrated to be a promising mutual-adaptive solution for low thermal conductivity and high scalability[3-8].

While thermal resistance at nanocrystalline grain boundaries plays a critical role for reducing thermal conductivity, little is known in experiment about to what extent the boundaries inhibit thermal transport, because thermal characterization of nanocrystalline thermoelectrics has been limited to measurements of effective thermal conductivity of the entire composite. Although there have been several numerical studies discussing causal relation between atomistic structure of a nanocrystalline grain boundary and its thermal boundary conductance (TBC) or resistance (TBR) $[9,10]$, the modeling has been 
limited to ideal interfaces and is not sufficient to rigorously characterize complex boundary structures that arise in actual high-energy processes such as sintering. Therefore, an experiment to directly measure $\mathrm{TBC}$ of representative sintered interfaces is needed to understand the controllability of TBC by interface nanostructures. Such knowledge will open up the possibility to truly engineer phonon transport to further improve $Z T$ of nanocrystalline thermoelectric materials.

Among various methods to measure thermal transport across solid interfaces, time-domain thermoreflectance method (TDTR)[11] utilizing transient reflectivity change has been widely used to measure TBCs in thin layered structures[12-14]. However, the method has not been applied to directly-bonded interfaces such as a sintered interface because the method is only applicable to highly planar and uniform interface located within thermal penetration depth from the heated surface (typically up to $\mu \mathrm{m}$ order). We have thus developed a process to fabricate a TDTR-measurable sample consisting of a silicon ( $\mathrm{Si}$ ) thin-film and Si substrate directly-bonded with highly planar and uniform interface. Here, we report TBC of sintered Si-Si interface, which serves as a model system for the interfaces in a sintered nanocrystalline silicon. The choice of material is encouraged by the recent progress in $\mathrm{Si}$ nanocrystalline thermoelectrics[5,15], whose highest $Z T$ is currently about 0.5 at $900 \mathrm{~K}$. Further improvement of $Z T$ would make Si-based thermoelectric a strong candidate for practical use with its high abundance, environmental friendliness, safety, and thermal stability.

By performing TDTR measurements of interfaces sintered at different temperatures, we identify the dependence of TBC on the interface structures. Here, we pay particular attention to the silicon oxide $\left(\mathrm{SiO}_{x}\right)$ nanostructures at the interface. $\mathrm{SiO}_{x}$ structures have 
been observed to precipitate at grain boundaries of sintered nanocrystalline silicon [15-17], and thus is a variable control parameter in the actual development of Si-based thermoelectrics. Furthermore, by combining the experimental results with a theoretical calculation that relates the value of TBC to the effective thermal conductivity of bulk nanocrystalline silicon, the current work reveals a great controllability of the effective thermal conductivity by interface structure from microscopic viewpoint.

\section{Experimental method}

A schematic diagram of the sample fabrication is shown in Fig. 1. We have developed a process to fabricate a thin-Si/Si-substrate bonded structure that meets the above geometrical requisites by utilizing an SOI wafer and selective chemical etching. An SOI wafer consists of highly parallel and smooth three layers: thin-Si $(\mathrm{SOI}) /$ thin $-\mathrm{SiO}_{2}(\mathrm{BOX}) /$ handle. As in Fig. 1, an SOI wafer and a Si wafer are cleaned with RCA and baked at $500{ }^{\circ} \mathrm{C}$ before bonding. After bonding, the handle Si and BOX were removed by etching with potassium hydrate $(\mathrm{KOH})$ and hydrofluoric acid (HF), respectively, to obtain a thin-Si/Si-wafer structure.

For sintering, we used plasma activated sintering (PAS) (also known as spark plasma sintering) technique, which can effectively raise temperature and bond interfaces of materials by applying pulsed/direct current and mechanical pressure in vacuum[18]. As nano-powders are sintered inside a carbon (graphite) mold in a common fabrication process of nanocrystalline thermoelectrics, we placed $12 \times 12 \mathrm{~mm}^{2} \mathrm{SOI}\left(\mathrm{Si} 292 \mathrm{~nm} / \mathrm{SiO}_{2}\right.$ $400 \mathrm{~nm} / \mathrm{Si} 675 \mu \mathrm{m}$ ) and Si chip face-to-face in the carbon mold. In the sintering process, we increased the temperature to the target value in 5 minutes and decreased it to 
ambient temperature in 7 minutes, applying constant pressure of $42 \mathrm{MPa}$ and pulsed current for the initial 30 seconds followed by direct current. The interface structure was varied by changing the maximum sintering temperature $\left(750{ }^{\circ} \mathrm{C} \sim 1100{ }^{\circ} \mathrm{C}\right)$ and the surface treatment of SOI and Si wafer before sintering. As for the surface treatment, we etched away the native oxide layers of SOI and $\mathrm{Si}$ wafer by HF for some samples to investigate the influence of the residual oxide layers. In addition, the matching of the crystal plane orientations was varied from $\mathrm{SOI}<100>/ \mathrm{Si}<100>$ to $\mathrm{SOI}<100>/ \mathrm{Si}<111>$ to investigate the effect of lattice mismatch at the interface.

With the fabricated samples, TBC across the interface can be measured with TDTR. A Ti: Sapphire femtosecond pulsed laser (wave length: $800 \mathrm{~nm}$, pulse width: 100 fs and pulse rate: $80 \mathrm{MHz}$ ) is split into pump and probe. The pump beam, modulated at 11.05 $\mathrm{MHz}$, is converted into blue beam (400 nm) with BIBO crystal, and heats the aluminum (Al) layer on the sample (Fig. 1). The arrival timing of the probe beam is adjusted by a mechanical delay stage. The pump and probe $1 / e^{2}$ radii at the sample surface are $30 \mu \mathrm{m}$ and $5 \mu \mathrm{m}$, respectively. The aluminum acts as a heater, which absorbs pump energy within several nanometers from the surface, and as a thermometer with relatively large thermoreflectance. The aluminum films was deposited using electron beam evaporation right after the selective chemical etching. Simultaneously, we also deposit aluminum on bare HF-etched $\mathrm{Si}$ wafer to determine in advance $\mathrm{TBC}$ of $\mathrm{Al}-\mathrm{Si}$ interface and thickness of Al film with TDTR. The measured TBC $\left(3.3 \times 10^{2} \mathrm{MWm}^{-2} \mathrm{~K}^{-1}\right)$ agreed well with the reported value $\left(3.5 \mathrm{MWm}^{-2} \mathrm{~K}^{-1}\right)$ [19], and the thickness of $\mathrm{Al}$ film was verified with the transmission electron microscopy (TEM). 
The ratio of the in-phase signal to out-of-phase lock-in-signals $(-X / Y)$ at the modulation frequency is measured, and the data from 200 ps to 1 ns were fitted by the solution of axisymmetric heat conduction equations for a layered structure model[20]. By fixing the thermal conductivity of thin Si to that of bulk, the remaining fitting parameter is only TBC of Si-Si interface. Although we could have taken the size effect of Si thermal conductivity into account, which was measured to reduce the value to about $120 \mathrm{Wm}^{-1} \mathrm{~K}^{-1}$, it was found to make negligible difference (within the measurement uncertainty) in the TBC values.

\section{Results and discussions}

The structure of $\mathrm{Si}-\mathrm{Si}$ sintered interface was characterized by TEM. As seen in the cross-sectional TEM of the interface sintered at $1100{ }^{\circ} \mathrm{C}$ without $\mathrm{HF}$ pretreatment (Fig. 2), all the samples were fabricated with high planarity and uniformity. In case of $\mathrm{SOI}<100>/ \mathrm{Si}<100>$ interfaces without $\mathrm{HF}$ pretreatment, an amorphous interlayer was observed, whose composition was identified to be $\mathrm{SiO}_{x}$ (oxygen concentration: 40 at. $\%$ ) by the energy dispersive X-ray spectroscopy (EDS) analysis. The amorphous $\mathrm{SiO}_{x}$ $\left(\mathrm{a}-\mathrm{SiO}_{x}\right)$ interlayer is attributed to the native oxide on the surfaces of both SOI and $\mathrm{Si}$ wafers. As seen in the high-resolution TEM (HR-TEM) images, the a-SiO $\mathrm{S}_{x}$ interlayer is a $2.4 \mathrm{~nm}$-thick uniform layer in case of $1100{ }^{\circ} \mathrm{C}$ (Fig. 3a), and is $3 \mathrm{~nm}$-thick layer with fragmentary line defects of $50 \%$ occupancy in case of $750{ }^{\circ} \mathrm{C}$ (Fig. 3b).

Figure 4 shows the TBC of the interfaces measured by TDTR. Together with the TEM images, the general conclusion is that the sintering temperature has minor influence on the $\mathrm{a}-\mathrm{SiO}_{x}$ interlayer structure, and thus on TBC. Quantitatively, the TBC 
in case of $1100{ }^{\circ} \mathrm{C}$ agrees well with the total conductance of $\mathrm{Si} / \mathrm{SiO}_{2}(2.2 \mathrm{~nm}) / \mathrm{Si}$ structure calculated by using phonon wave packet dynamics (PWD) $\left(3.1 \times 10^{2} \mathrm{Wm}^{-2} \mathrm{~K}^{-1}\right)$ and non-equilibrium molecular dynamics (NEMD) $\left(2.9 \times 10^{2} \quad \mathrm{Wm}^{-2} \mathrm{~K}^{-1}\right)[21]$. Such interlayer thermal resistance can be considered as a sum of the thermal resistances of two $\mathrm{Si} / \mathrm{SiO}_{x}$ interfaces and that of $\mathrm{a}_{-} \mathrm{SiO}_{x}$ itself. Then, by assuming the thermal conductivity of the $\mathrm{a}_{-} \mathrm{SiO}_{x}$ to lie in between the bulk values of a-SiO${ }_{2}$ (1.4 $\left.\mathrm{Wm}^{-1} \mathrm{~K}^{-1}\right)[22,23]$ and $\mathrm{a}-\mathrm{Si}\left(1.8 \mathrm{Wm}^{-1} \mathrm{~K}^{-1}\right)[24,25]$, the thermal conductance of a $\mathrm{Si} / \mathrm{SiO}_{x}$ interface can be calculated to be $7.0 \sim 8.5 \times 10^{2} \mathrm{MWm}^{-2} \mathrm{~K}^{-1}$. This is similar to the TBC of various $\mathrm{Si} / \mathrm{SiO}_{2}$ interfaces reported both numerically and experimentally. Deng et al. calculated TBC of $\mathrm{c}-\mathrm{SiO}_{2} / \mathrm{Si}$ to be $6.8 \times 10^{2} \mathrm{MWm}^{-2} \mathrm{~K}^{-1}$ and $7.3 \times 10^{2} \mathrm{MWm}^{-2} \mathrm{~K}^{-1}$ using NEMD and PWD.[21] Chen et al. calculated TBC of a strongly coupled a- $\mathrm{SiO}_{2} / \mathrm{Si}$ system to be $1.1 \times 10^{3} \mathrm{MWm}^{-2} \mathrm{~K}^{-1}$ using NEMD simulation[26]. Finally, Hurley et al. measured the value of $\mathrm{Si} / \mathrm{SiO}_{2}$ in bicrystal $\mathrm{Si}$ to range from $4.2 \times 10^{2}$ to $6.7 \times 10^{2}$ $\mathrm{MWm}^{-2} \mathrm{~K}^{-1} \cdot[27]$

The above results on the $\mathrm{TBC}$ of uniform $\mathrm{a}-\mathrm{SiO}_{x}$ layer confirm the accuracy of the current measurements. Turning now to 3 nm-thick a-SiO ${ }_{x}$ (Fig. 3b), the measured TBC of the sample sintered at $750{ }^{\circ} \mathrm{C}\left(1.6 \times 10^{2} \mathrm{MWm}^{-2} \mathrm{~K}^{-1}\right)$ is $20 \%$ smaller than $2.1 \times 10^{2}$ $\mathrm{MWm}^{-2} \mathrm{~K}^{-1}$ estimated from the above values of a-SiO${ }_{\mathrm{x}}$ thermal conductivity and $\mathrm{Si} / \mathrm{SiO}_{x}$ thermal conductance. This suggests that the line defects observed in Fig. 3b inhibit the thermal transport. If we assume that the total $\mathrm{TBC}$ is weighted average of the $\mathrm{TBC}$ of non-defect $\left(2.1 \times 10^{2} \mathrm{MWm}^{-2} \mathrm{~K}^{-1}\right)$ and defect region based on their occupancy at the interface, the TBC of the line defect is calculated to be $1.1 \times 10^{2} \mathrm{MWm}^{-2} \mathrm{~K}^{-1}$. 
On the other hand, the interfaces pretreated with $\mathrm{HF}$ have no a-SiO $\mathrm{x}_{\mathrm{x}}$ interlayer and their structures greatly depend on the sintering temperature. In case of the highest sintering temperature, $1100{ }^{\circ} \mathrm{C}$, pure $\mathrm{Si}-\mathrm{Si}$ interface is formed with lattice deformation and local strains (Fig. 3c). However, despite the lattice deformation, we find that TBC is larger than $10 \mathrm{GW} / \mathrm{m}^{2} \mathrm{~K}$, which is more than $10 \%$ of thermal conductance of single crystal Si of a few nanometer thick. The reason for the observed relatively large TBC may be that the lattice and crystal orientation of deformed region is still continuous, and that the strain is less than a few percent, which is expected to have limited impact on heat conduction[28]. In case of $950{ }^{\circ} \mathrm{C}$ sintering temperature, precipitate-like $\mathrm{SiO}_{x}$ nanostructures with 3 at. \% oxygen in average were observed (Fig. 3d). The nominal size of nanostructures is about $15 \mathrm{~nm} \times 10 \mathrm{~nm}$ (in the direction parallel and normal to the interface, respectively), and they occupy about $40 \%$ of the interface area. The HR-TEM images revealed that they have crystalline structures, although the lattices are likely to be deformed since the solubility limit of oxygen in silicon crystal is ppm order.[29] The presence of the crystalline $\mathrm{SiO}_{x}\left(\mathrm{c}-\mathrm{SiO}_{x}\right)$ nanostructures greatly reduces the TBC but the value is still as high as $9.2 \times 10^{2} \mathrm{MWm}^{-2} \mathrm{~K}^{-1}$. The $\mathrm{TBC}$ can be further reduced to $4.4 \times 10^{2} \mathrm{MWm}^{-2} \mathrm{~K}^{-1}$ by lowering the sintering temperature to $750{ }^{\circ} \mathrm{C}$ (Fig. 3e), where $\mathrm{c}^{-\mathrm{SiO}_{x}}$ nanostructures are smaller $(6 \mathrm{~nm} \times 3 \mathrm{~nm})$ and the oxygen concentration is somewhat larger (average 7 at. \%).

The formation of $\mathrm{c}-\mathrm{SiO}_{x}$ nanostructures can be originated from small amount of oxygen adsorbed during the sample preparation for the sintering process. Firstly, right after the HF etching of native oxide, oxide layer regrowth $(\sim 2 \AA)[30]$ during rinsing with ultrapure water and exposure to ambient air till the sintering. Note that the amount of oxygen atoms of the $\mathrm{SiO}_{x}$ nanostructures approximately correspond to $2 \AA$-thick-SiO 
with 40 at. $\%$ oxygen. The oxide layer is then locally broken down by the kA-order-current of plasma sintering, making the distribution of oxygen discontinuous, and small amount of oxygen thermally diffuse into the crystalline lattice near the surface. The size of the nanostructure increases with the kinetic energy and thus with temperature.

So far, we have discussed the interface between surfaces with the same crystal orientation in order to independently characterize the influence of the $\mathrm{SiO}_{x}$ nanostructures. However, in the actual nanocrystalline materials formed by sintering, crystalline orientation of the nanoparticles are usually distributed randomly, and the resulting lattice mismatch is expected to be an additional source of thermal resistance. This can be confirmed in the case of $\mathrm{SOI}<100>/ \mathrm{Si}<111>$ with the HF pretreatment shown in Fig. 4, where TBC becomes significantly smaller than $\mathrm{SOI}<100>/ \mathrm{Si}<100>$ at any sintering temperature. In the $1100{ }^{\circ} \mathrm{C}$ case with absence of $\mathrm{SiO}_{x}, \mathrm{TBC}$ is measured to be $1.7 \mathrm{GWm}^{-2} \mathrm{~K}^{-1}$. These values are on the same order with the previously reported values obtained by molecular dynamics simulations of Si-Si twist boundary, where two $<111>$ surfaces of Si crystal are connected and twisted against each other.[9,10] It was reported that TBC of the twist boundary is on the order of $1 \mathrm{GWm}^{-2} \mathrm{~K}^{-1}$, and decreases with increasing boundary energy, i.e. increasing degree of lattice mismatch. The mismatch between $\langle 100\rangle$ and $\langle 111\rangle$ in this experiment is expected to be larger than any of the twist boundaries because the surface lattice structures of $\langle 100\rangle$ and $\langle 111\rangle$ are respectively square lattice and hexagonal lattice, and no corresponding lattice points exist at the interface. 
Oxygen atoms were also detected at the $\mathrm{SOI}<100>/ \mathrm{Si}<111>$ interface by EDS analysis. In case of the lowest sintering temperature, $810^{\circ} \mathrm{C}$, the interface structure is

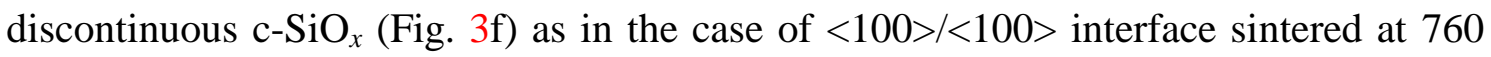
${ }^{\circ} \mathrm{C}$ (Fig. 3e), although the crystallinity for this case may be less evident from the TEM image due to mixture with the lattice mismatch. It is interesting that $\mathrm{TBC}$ of the discontinuous c-SiO $x$ structures is as small as the uniform a-SiO $x$ structures (Fig. 3a) despite that the former $(\sim 1 \mathrm{~nm})$ is much thinner that the latter $(\sim 3 \mathrm{~nm})$. The concentration of oxygen in the $\mathrm{SiO}_{x}$ is 14 at. \%, which is consistent with the amount of oxygen in $2 \AA$-thick native oxide. These suggest that contribution of local crystal c-SiO structure and lattice mismatch is far more efficient than the a-SiO ${ }_{x}$ structure even with smaller oxygen concentration.

In case of $\mathrm{SOI}<100>/ \mathrm{Si}<111>$ without the $\mathrm{HF}$ pretreatment, the $\mathrm{TBC}$ value is almost the same as the case with $\mathrm{SOI}<100>/ \mathrm{Si}<100>$, indicating that thermal resistance is dominated by the intrinsic phonon scattering in the uniform $\mathrm{a}-\mathrm{SiO}_{\mathrm{x}}$ interlayer.

We now evaluate the impact of the TBC reduction on thermal conductivity of a nanocrystalline material assuming that the same TBC reduction occurs at all the sintered grain boundaries. We start by estimating the phonon transmission probability $t$ at the sintered Si-Si interfaces. From Landauer formula, TBC can be expressed as[31]

$$
G=\frac{\sigma}{1-\sigma / \sigma_{a}}
$$

where $\sigma$ and $\sigma_{a}$ are

$$
\sigma=\frac{1}{2 V} \sum_{\mathbf{k}, s} \hbar \omega \frac{\partial n_{\mathrm{B}}}{\partial T} v_{x} t
$$




$$
\sigma_{a}=\frac{1}{2 V} \sum_{\mathbf{k}, s} \hbar \omega \frac{\partial n_{\mathrm{B}}}{\partial T} v_{x}
$$

Here, $\omega$ is frequency, $n_{\mathrm{B}}$ is Bose-Einstein distribution, $v_{x}$ is $x$ component of group velocity, and $V$ is crystal volume. Indices $\mathbf{k}$ and $s$ denote the wave vector and branch. Therefore, the phonon transmission probability can be obtained by knowing phonon frequencies and group velocities.

The frequency dependence of the transmission probability is modeled as[35,36],

$$
t(\omega)=\frac{1}{\gamma \omega / \omega_{\max }+1}
$$

with fitting parameter $\gamma$ and maximum frequency $\omega_{\max }=96 \mathrm{THz}$. The function returns 1 when $\omega=0$ and monotonically decreases with increasing $\omega$. This trend agrees with that of more rigorous calculations of an interface between dissimilar materials using Green's function method[37] and twisted grain boundaries using PWD.[9,10] For the measured TBC values, we obtained $\gamma$ and $t$ as summarized in Table 1 .

Thermal conductivity $\kappa$ can be obtained as an analytical solution of Boltzmann transport equation,

$$
\kappa=\frac{1}{3} \sum_{s} \int_{\omega} D C v_{s} \Lambda d \omega
$$

where $D$ is phonon density of state, $C$ is specific heat, and $\Lambda$ is mean free path, respectively. $\Lambda$ of a nanostructure is calculated by applying Matthiessen's rule to mean free paths of phonon-phonon scattering $\left(\Lambda_{\text {bulk }}\right)$ and phonon-boundary scattering $\left(\Lambda_{\text {bdy }}\right)$.

$$
\Lambda^{-1}(\omega, s)=\Lambda_{\text {bulk }}^{-1}(\omega, s)+\Lambda_{\text {bdy }}^{-1}(\omega, s) .
$$


The bulk phonon transport properties $D, C, v$, and $\Lambda_{\text {bulk }}\left(=v \tau_{\text {bulk }}\right)$ were obtained by harmonic and anharmonic lattice dynamics with the first-principle-based interatomic force constants $[33,34]$, where $\tau_{\text {bulk }}$ is the phonon relaxation time. The harmonic lattice dynamics calculations were performed on a wave-vector mesh of $512 \times 512 \times 512$ to obtain the frequency dependences of $D, C$, and $v$. On the other hand, the anharmonic lattice dynamics calculations used to obtain $\tau_{\text {bulk }}$ is more computationally intensive. Therefore, the calculations were performed for $30 \times 30 \times 30$ wave-vector mesh, and the obtained values were fitted with a power-law function for each branch in the low frequency regime, where the data points are relatively coarse. The obtained frequency-dependent bulk phonon transport properties have been confirmed to reproduce the bulk thermal conductivity of Si crystal.

By modeling the nanocrystalline structure as a densely packed simple cubic grains of grain size $L, \Lambda_{\text {bdy }}$ of a nanocrystalline structure were calculated as,

$$
\frac{\Lambda_{\text {bdy }}}{L}=\left[\left(\frac{\frac{3}{4} t}{1-t}\right)^{-1}+1.12^{-1}\right]^{-1}
$$

which is a Matthieesen's rule combining the mean free path of superlattice (first term on RHS) [38] and square nanowire (second term on RHS) [39] assuming diffuse scattering at the boundaries. The diffuse scattering should be an reasonable assumption for interfaces with nanoscale structures (Fig. 3). Here, the grain size distribution is approximated by a single value of $L$, which was recently found to be valid for a lognormal distribution at least up to standard deviation of 0.35 [40]. 
The calculation obtains dependence of nanocrystalline thermal conductivity on the grain size and TBC. Fig. 5 shows the results in case of $L=20 \mathrm{~nm}, 50 \mathrm{~nm}$, and $100 \mathrm{~nm}$ calculated based on the TBCs of HF-treated samples. In the case of $\mathrm{SOI}<100>/ \mathrm{Si}<111>$ sintered at $810{ }^{\circ} \mathrm{C}$ with $\mathrm{HF}$ pretreatment, where fragmental c-SiO$x$ structure with crystal orientation mismatch at the interface reduce $\mathrm{TBC}$ to $1.9 \times 10^{2} \mathrm{MWm}^{-2} \mathrm{~K}^{-1}$, the calculated nanocrystalline lattice thermal conductivity reaches 13,7 , and $3 \mathrm{Wm}^{-1} \mathrm{~K}^{-1}$ at room temperature with the grain size of $100 \mathrm{~nm}, 50 \mathrm{~nm}$, and $20 \mathrm{~nm}$, respectively. These predicted thermal conductivities are significantly smaller than that of undoped nano-bulk Si reported by Bux et al. $\left(48 \mathrm{Wm}^{-1} \mathrm{~K}^{-1}\right)[5]$, where feature grain size is $20 \mathrm{~nm}$ with a distribution from nanocrystalline domain $(10-15 \mathrm{~nm})$ to grown grain domain (50-100 nm), and even smaller than that of relatively high porosity (17\%) pure nanocrystalline reported by Wang et al. $\left(9 \mathrm{Wm}^{-1} \mathrm{~K}^{-1}\right)[35]$, where the mean grain diameter is $64 \mathrm{~nm}$. Note that the thermal conductivity in the case of $20 \mathrm{~nm}$ is approaching the value of bulk amorphous Si [24,25]. This fragmental structure can be also advantageous for electrical transport. While the locally fragmental c- $\mathrm{SiO}_{x}$ structure is expected to be less influential for electrons conduction than phonons because the $\mathrm{c}-\mathrm{SiO}_{\mathrm{x}}$ structure is highly crystalline with small oxide concentration, and the $\mathrm{Si}$ channels between the $\mathrm{c}-\mathrm{SiO}_{\mathrm{x}}$ structures allow electron wave functions to be coherent across the interface.[41] In view of particle transport, electrons, which has shorter mean free path than phonons[42], are expected to be less affected by the fragmental nanoscale obstacles.

\section{Conclusions}


In summary, we have developed a process to fabricate a highly planar and uniform bonded-interface between $\mathrm{Si}$ thin film and $\mathrm{Si}$ substrate utilizing $\mathrm{SOI}$ and selective chemical etching, and realized TBC measurements on sintered interfaces with TDTR. We have firstly found that sintering temperature and pre-removal of native oxide with $\mathrm{HF}$ alters the interface structure from uniform amorphous to local crystalline $\mathrm{SiO}_{x}$ structure. We verified that the $\mathrm{TBC}$ of uniform-amorphous $\mathrm{SiO}_{x}(\mathrm{O}: 40$ at.\%) interlayer is consistent with the previous numerical and experimental studies.[21,26,27] The nanometer-scale crystalline $\mathrm{SiO}_{x}$ structures greatly reduce $\mathrm{TBC}$ despite the low oxygen concentration and high crystallinity. The reduction becomes even stronger and TBC reaches $1.9 \times 10^{2} \mathrm{MWm}^{-2} \mathrm{~K}^{-1}$ by introducing crystal orientation mismatch. The analytical calculation reveals that the $\mathrm{TBC}$ is small enough to reduce thermal conductivity of nanocrystalline Si to 7 and $3 \mathrm{Wm}^{-1} \mathrm{~K}^{-1}$ for the grain sizes of $50 \mathrm{~nm}$ and $20 \mathrm{~nm}$, which are considerably smaller than the previously reported values of undoped nanocrystalline $\mathrm{Si}$ [5,35], and even approaching the value of bulk amorphous $\mathrm{Si}$ [24,25]. The discontinuous structure of $\mathrm{c}-\mathrm{SiO}_{\mathrm{x}}$ is expected to have advantage also in electric transport because of the highly crystalline structure with relatively small oxygen content and coherent silicon channels between the structures. Current work provides fundamental knowledge in how and to what extent TBC varies with sintered interface structure, and how much the TBC reduction affects thermal conductivity of nanocrystalline $\mathrm{Si}$, which is useful to truly engineer thermal transport across the sintered interface for further $Z T$ enhancement.

\section{Acknowledgements}


This work was partially supported by Japan Science and Technology Agency PRESTO, and KAKENHI 26709009 and 26630061.

\section{References}

[1] R. Venkatasubramanian, E. Siivola, T. Colpitts, B. O’Quinn, Nature 413 (2001) 597.

[2] T.C. Harman, P.J. Taylor, M.P. Walsh, B.E. LaForge, Science 297 (2002) 2229.

[3] B. Poudel, Q. Hao, Y. Ma, Y. Lan, A. Minnich, B. Yu, X. Yan, D. Wang, A. Muto, D. Vashaee, X. Chen, J. Liu, M.S. Dresselhaus, G. Chen, Z. Ren, Science. 320 (2008) 634.

[4] Y. Lan, B. Poudel, Y. Ma, D. Wang, M.S. Dresselhaus, G. Chen, Z. Ren, Nano Lett. 9 (2009) 1419.

[5] S.K. Bux, R.G. Blair, P.K. Gogna, H. Lee, G. Chen, M.S. Dresselhaus, R.B. Kaner, J.P. Fleurial, Adv. Funct. Mater. 19 (2009) 2445.

[6] X. Yan, G. Joshi, W. Liu, Y. Lan, H. Wang, S. Lee, J.W. Simonson, S.J. Poon, T.M. Tritt, G. Chen, Z.F. Ren, Nano Lett. 11 (2011) 556.

[7] H. Alam, S. Ramakrishna, Nano Energy 2 (2013) 190.

[8] W. Liu, X. Yan, G. Chen, Z. Ren, Nano Energy 1 (2012) 42.

[9] S. Ju, X. Liang, J. Appl. Phys. 113 (2013) 053513.

[10] P.K. Schelling, S.R. Phillpot, P. Keblinski, J. Appl. Phys. 95 (2004) 6082 .

[11] C.A. Paddock, G.L. Eesley, J. Appl. Phys. 60 (1986) 285.

[12] M.D. Losego, M.E. Grady, N.R. Sottos, D.G. Cahill, P. V Braun, Nat. Mater. 11 (2012) 502. 
[13] R. Costescu, M. Wall, D.G. Cahill, Phys. Rev. B 67 (2003) 054302.

[14] P.E. Hopkins, J.C. Duda, C.W. Petz, J. a. Floro, Phys. Rev. B Condens. Matter Mater. Phys. 84 (2011) 035438.

[15] N. Petermann, N. Stein, G. Schierning, R. Theissmann, B. Stoib, M.S. Brandt, C. Hecht, S. Christof, H. Wiggers, J. Phys. D 44 (2011) 174034.

[16] G. Schierning, R. Theissmann, N. Stein, N. Petermann, A. Becker, M. Engenhorst, V. Kessler, M. Geller, A. Beckel, H. Wiggers, R. Schmechel, J. Appl. Phys. 110 (2011) 113515.

[17] T. Claudio, G. Schierning, R. Theissmann, H. Wiggers, H. Schober, M.M. Koza, R.P. Hermann, J. Mater. Sci. 48 (2013) 2836.

[18] Z.A. Munir, U. Anselmi-Tamburini, M. Ohyanagi, J. Mater. Sci. 41 (2006) 763.

[19] A.J. Minnich, J.A. Johnson, A.J. Schmidt, K. Esfarjani, M.S. Dresselhaus, K.A. Nelson, G. Chen, Phys. Rev. Lett. 107 (2011) 095901.

[20] D.G. Cahill, Rev. Sci. Instrum. 75 (2004) 5119.

[21] B. Deng, A. Chernatynskiy, M. Khafizov, D.H. Hurley, S.R. Phillpot, J. Appl. Phys. 115 (2014) 084910.

[22] T. Yamane, N. Nagai, S. Katayama, M. Todoki, J. Appl. Phys. 91 (2002) 9772.

[23] K.T. Regner, D.P. Sellan, Z. Su, C.H. Amon, A.J.H. McGaughey, J. Malen, Nat. Commun. 4 (2013) 1640.

[24] H. Wada, T. Kamijoh, Jpn. J. Appl. Phys. 35 (1996) L648.

[25] T. Papa, F. Scudieri, M. Marinelli, U. Zammit, G. Cembali, Le J. Phys. Colloqes. 44 (1983) C5.

[26] J. Chen, G. Zhang, B. Li, J. Appl. Phys. 112 (2012) 064319. 
[27] D.H. Hurley, M. Khafizov, S.L. Shinde, J. Appl. Phys. 109 (2011) 083504.

[28] K.D. Parrish, A. Jain, J.M. Larkin, W. a. Saidi, A.J.H. McGaughey, Phys. Rev. B 90 (2014) 1.

[29] S. Messoloras, R.C. Newman, R.J. Stewart, J.H. Tucker, Semicond. Sci. Technol. 2 (1987) 14.

[30] M. Morita, T. Ohmi, E. Hasegawa, M. Kawakami, M. Ohwada, J. Appl. Phys. 68 (1990) 1272.

[31] Z. Tian, K. Esfarjani, G. Chen, Phys. Rev. B - Condens. Matter Mater. Phys. 89 (2014) 235307.

[32] G.P. Srivastava, The Physics of Phonons, A. Hilger, Bristol $\square$; Philadelphia, 1990.

[33] K. Esfarjani, H.T. Stokes, Phys. Rev. B - Condens. Matter Mater. Phys. 77 (2008) 144112.

[34] K. Esfarjani, G. Chen, H.T. Stokes, Phys. Rev. B - Condens. Matter Mater. Phys. 84 (2011) 085204.

[35] Z. Wang, J.E. Alaniz, W. Jang, J.E. Garay, C. Dames, Nano Lett. 11 (2011) 2206.

[36] C. Hua, A.J. Minnich, Semicond. Sci. Technol. 29 (2014) 124004.

[37] H. Zhao, J.B. Freund, J. Appl. Phys. 105 (2009) 013515.

[38] C. Dames, G. Chen, J. Appl. Phys. 95 (2004) 682.

[39] J.M. Ziman, Electrons and Phonons $\square$ : The Theory of Transport Phenomena in Solids, Clarendon Press $\square$; Oxford University Press, Oxford New York, 2001.

[40] T. Hori, J. Shiomi, C. Dames, J. Appl. Phys. (submitted). 
[41] Y. Nakamura, M. Isogawa, T. Ueda, S. Yamasaka, H. Matsui, J. Kikkawa, S. Ikeuchi, T. Oyake, T. Hori, J. Shiomi, A. Sakai, Nano Energy (2014) 1.

[42] B. Qiu, Z. Tian, A. Vallabhaneni, B. Liao, J.M. Mendoza, D. Oscar, arXiv:1409.4862. 
Table 1 The parameter $\gamma$ in Eq. (4) (transmission probability) obtained from measured TBC of samples.

\begin{tabular}{|c|c|c|c|c|}
\hline & $\begin{array}{l}\text { with or } \\
\text { without } \mathrm{HF}\end{array}$ & $\begin{array}{l}\text { Temperature } \\
{\left[{ }^{\circ} \mathrm{C}\right]}\end{array}$ & $\begin{array}{l}\text { Measured } G \\
\qquad\left[\mathrm{MW} / \mathrm{m}^{2} \mathrm{~K}\right]\end{array}$ & $\gamma$ \\
\hline \multirow[t]{5}{*}{$\mathrm{SOI}<100>/ \mathrm{Si}<100>$} & \multirow[t]{3}{*}{ Without } & 1100 & $2.4 \times 10^{2}$ & 11 \\
\hline & & 950 & $1.7 \times 10^{2}$ & 16 \\
\hline & & 750 & $1.6 \times 10^{2}$ & 17 \\
\hline & \multirow[t]{2}{*}{ With } & 950 & $9.2 \times 10^{2}$ & 2.3 \\
\hline & & 750 & $4.4 \times 10^{2}$ & 5.8 \\
\hline \multirow[t]{4}{*}{$\mathrm{SOI}<100>/ \mathrm{Si}<111>$} & Without & 1100 & $2.2 \times 10^{2}$ & 12 \\
\hline & \multirow[t]{3}{*}{ With } & 1100 & $1.7 \times 10^{3}$ & 1.4 \\
\hline & & 990 & $6.3 \times 10^{2}$ & 3.9 \\
\hline & & 810 & $1.9 \times 10^{2}$ & 14 \\
\hline
\end{tabular}




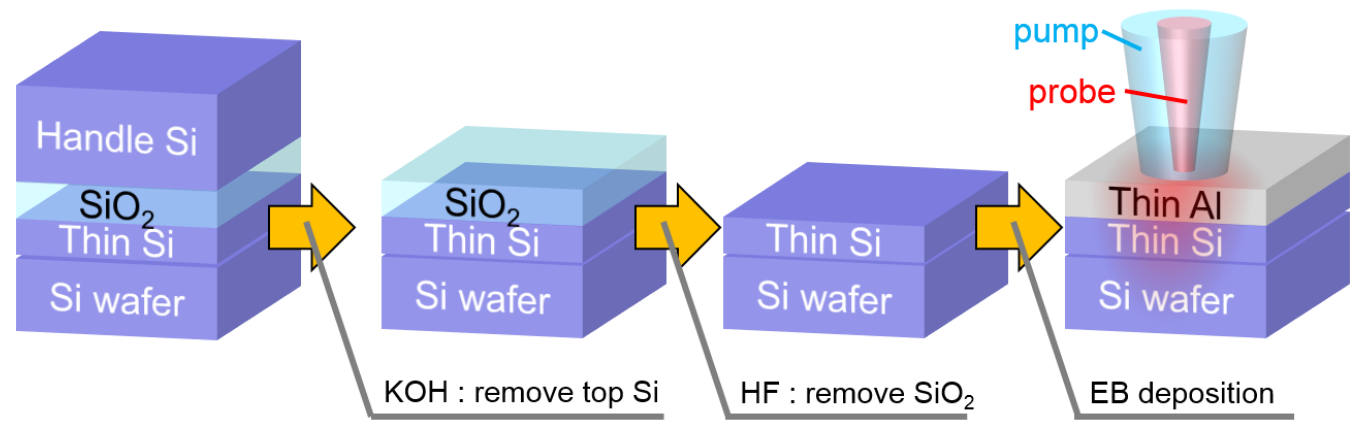

Figure 1. Schematic diagram of fabrication of thin-Si/Si-wafer bonded structure, and TDTR measurement. 


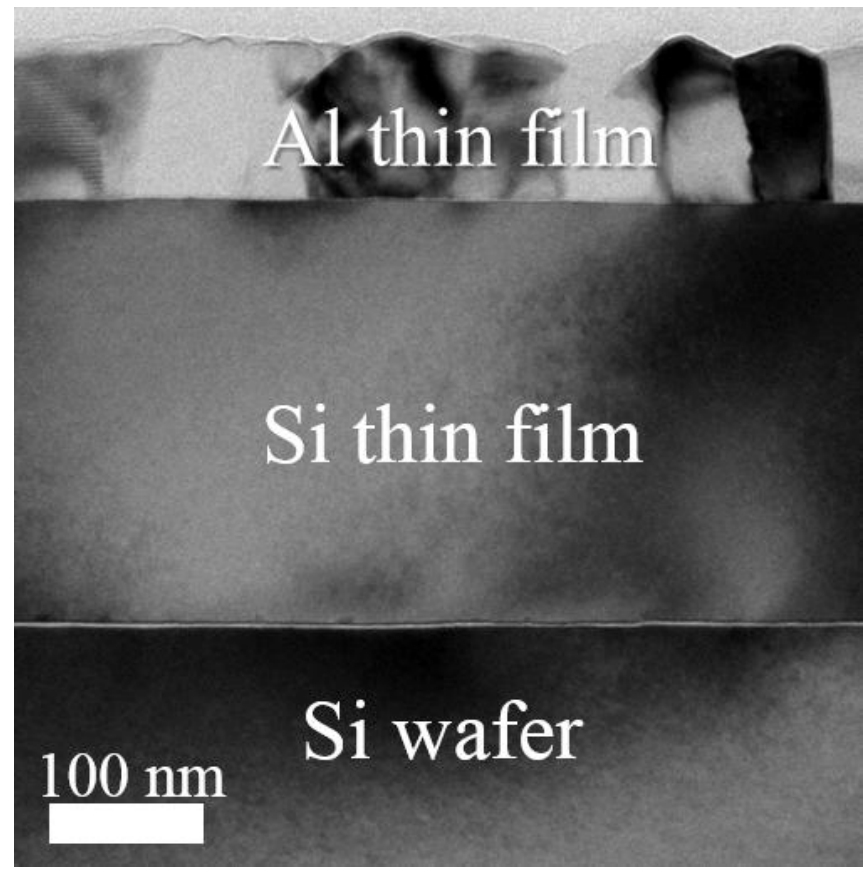

Figure 2. Cross-sectional TEM image of Si-Si structure sintered at $1100{ }^{\circ} \mathrm{C}$. 

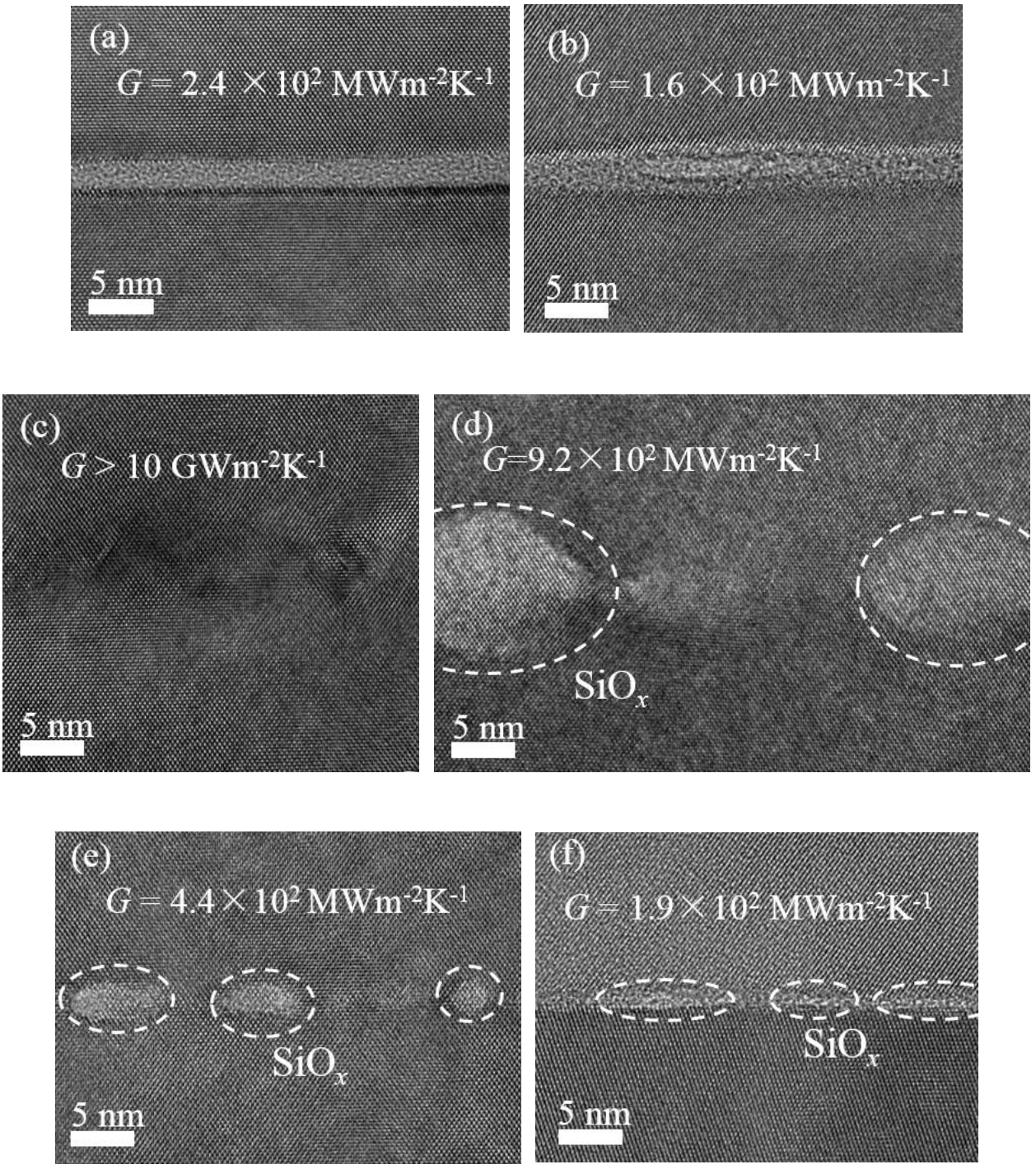

Figure 3. Cross sectional HR-TEM images of $\mathrm{SOI}<100>/ \mathrm{Si}<100>$ interfaces sintered at (a) 1100 ${ }^{\circ} \mathrm{C}$ and (b) $750{ }^{\circ} \mathrm{C}$ without HF pretreatment, $\mathrm{SOI}<100>/ \mathrm{Si}<100>$ interface sintered at (c) $1100{ }^{\circ} \mathrm{C}$, (d) $950{ }^{\circ} \mathrm{C}$ and (e) $750{ }^{\circ} \mathrm{C}$ with $\mathrm{HF}$ pretreatment, and $\mathrm{SOI}<100>/ \mathrm{Si}<111>$ interface sintered at 810 ${ }^{\circ} \mathrm{C}$ with $\mathrm{HF}$ pretreatment. $G\left[\mathrm{MWm}^{-2} \mathrm{~K}^{-1}\right]$ denotes total TBC of the interfaces. 


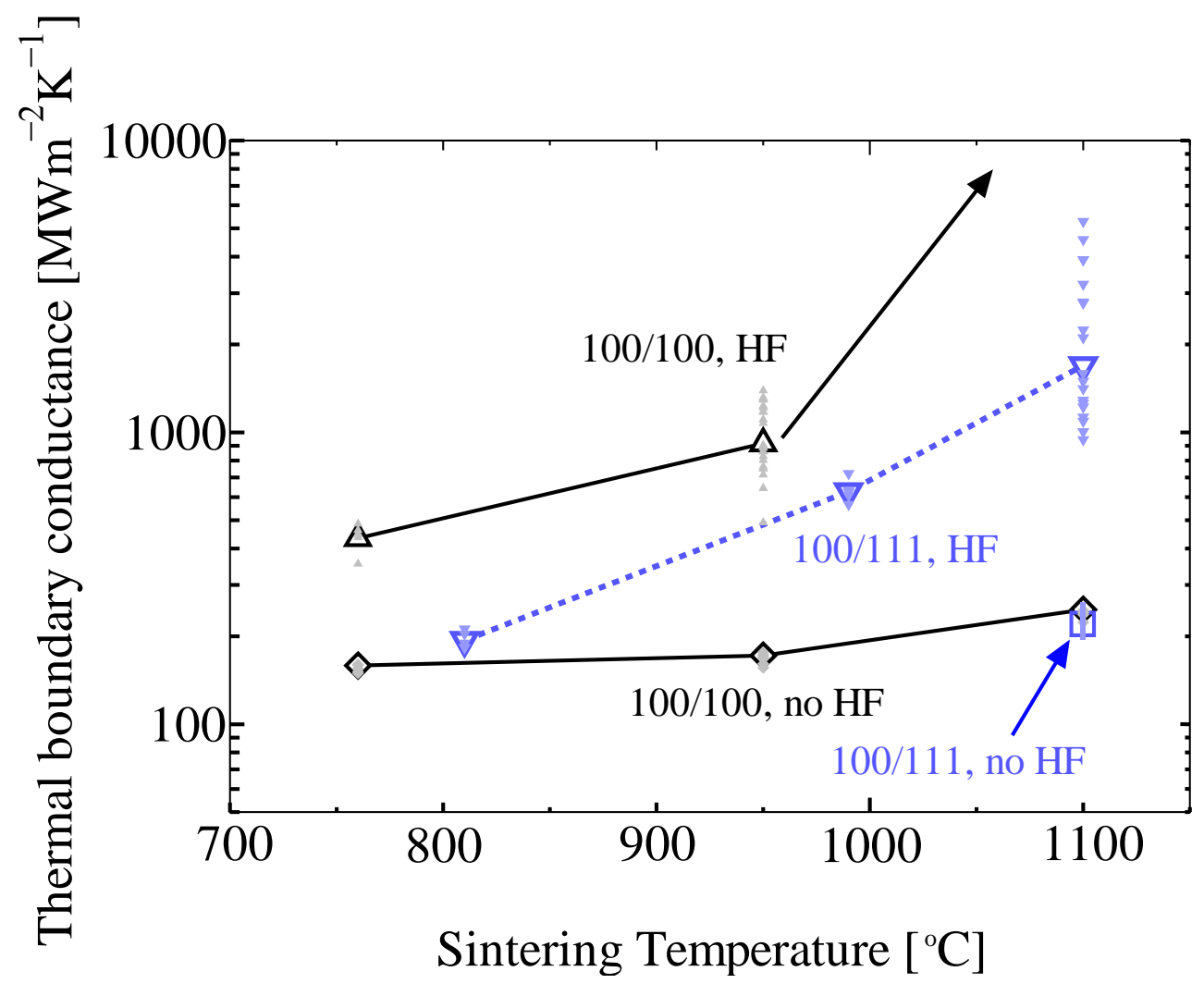

Figure 4. Measured thermal conductance of $\mathrm{Si}-\mathrm{Si}$ sintered interface as a function of sintering temperature for interfaces with different crystal orientations and surface pretreatments. For each sample, the measurements were performed at different locations and times on the surface. The averaged values are denoted with the larger markers, and the extent of data fluctuation is indicated by plotting all the data with smaller markers. 


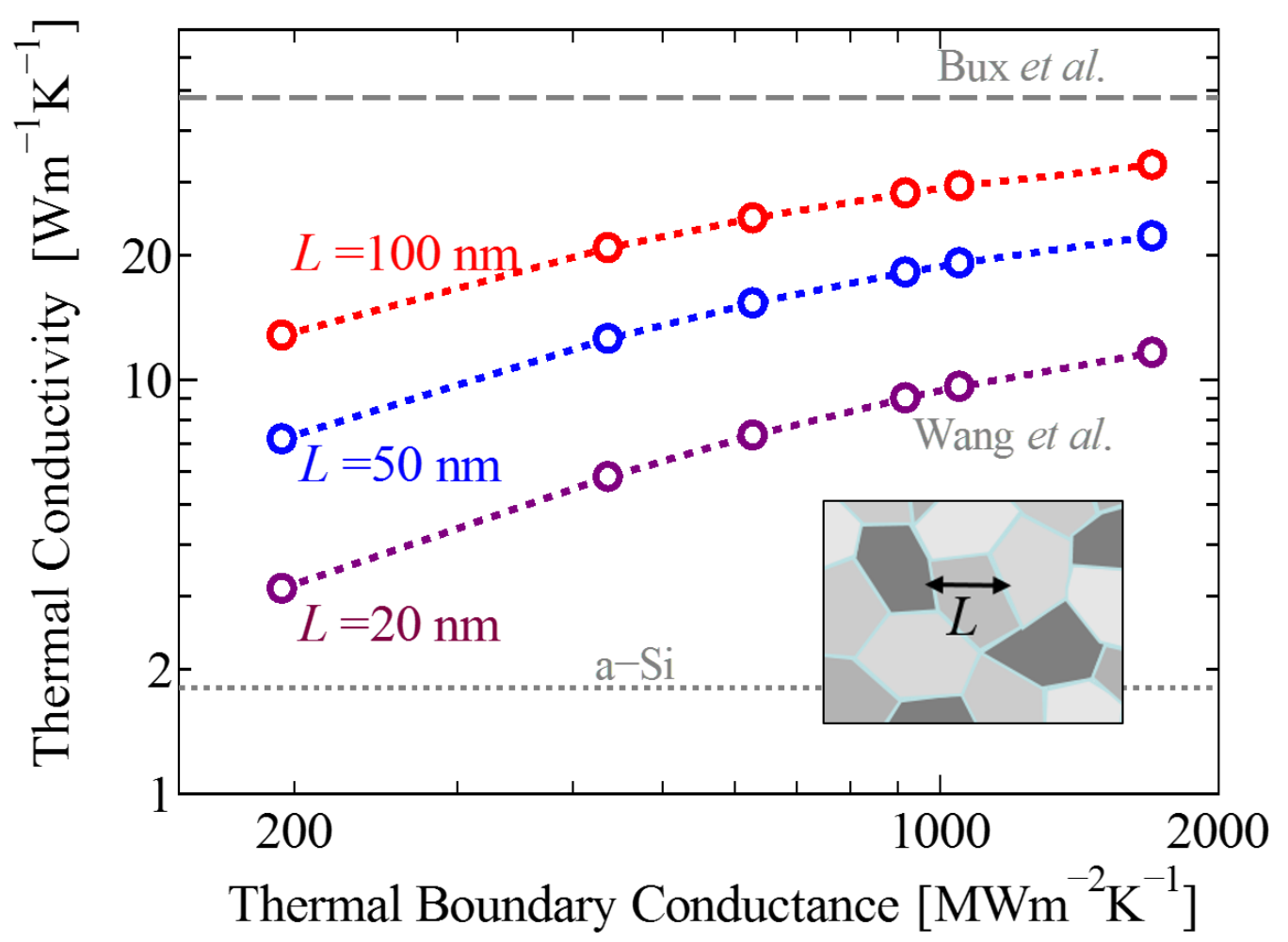

Figure 5. Modeled thermal conductivity of nanocrystalline with grain sizes $(L)$ of $20 \mathrm{~nm}, 50 \mathrm{~nm}$ and $100 \mathrm{~nm}$ as a function of thermal boundary conductance of Si-Si interface, compared with thermal conductivity of nanocrystalline Si reported by Bux et al. [5] and Wang et al. [35] (dashed lines) and that of a-Si[24,25] (dotted line). 
Personal portrait photo and a biosketch

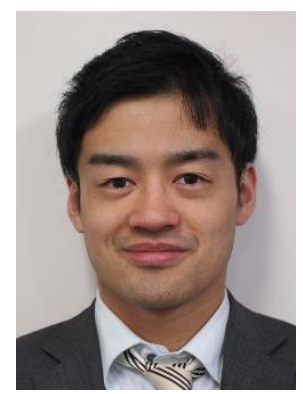

Masanori Sakata received his B. Eng. (2013) and M. Eng. (2015) in Mechanical Engineering from The University of Tokyo, Japan. His research interests focus on measurement of thermal transport across directly-bonded silicon interfaces, using optical pump-probe method. He is a recipient of Miura Award from Japanese Society of Mechanical Engineering.

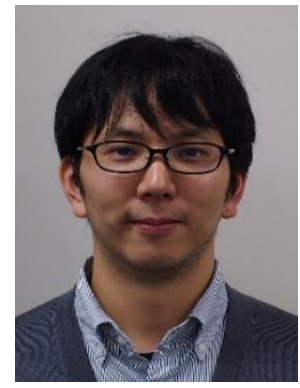

Takuma Hori received his B.S. (2010) and M.S.(2012) degrees in mechanical engineering from the University of Tokyo in Japan, and is currently a $\mathrm{PhD}$. student there. His research interest focuses on studies of phonon transport in nanoscale thermoelectric materials using numerical simulations.

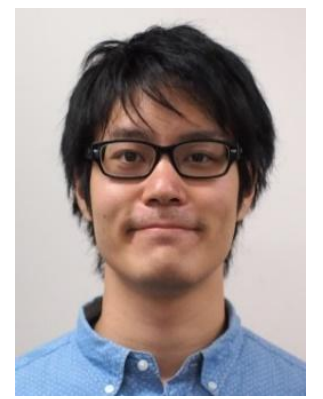

Takafumi Oyake received his B.Eng. (2012) and M. Eng. (2014) in Mechanical Engineering from The University of Tokyo, Japan. Currently, he is pursuing his Ph.D. there under Associate 
Professor Junichiro Shiomi. His research interests focus on experimental observations of thermal transport at nanoscale materials and interfaces, using optical experimental method.

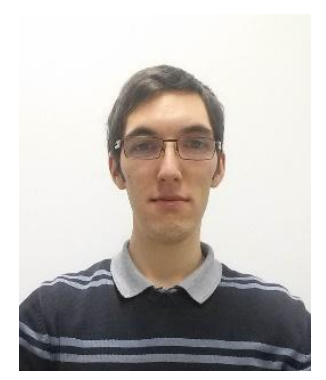

Jeremie Maire received is B. Eng. (2010) and M. Eng (2012) from Ecole Centrale Lyon, France where he is currently a $\mathrm{PhD}$, detached in LIMMS-CNRS/IIS at the Institute of Industrial Science, the University of Tokyo, under the supervision of Associate Professor Masahiro Nomura. His research interests focus on fabrication of Si-based nanostructures and experimental observations of thermal transport at the nanoscale using optical method.

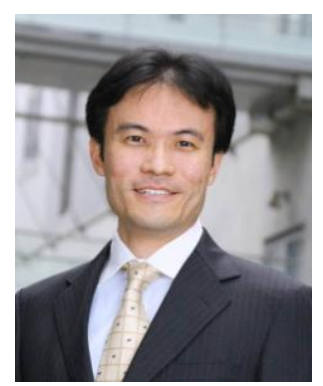

Masahiro Nomura received his B.E, M.E. and Ph. D. degrees in the applied physics all from the University of Tokyo, Japan, in 2000, 2002, and 2005, respectively. During 2004-2005, he was a Research Fellow of the Japan Society for the Promotion of Science. He joined Nanoelectronics Collaborative Research Center in 2005 and Institute for Nano Quantum Information Electronics, the University of Tokyo in 2007, as a Project Research Associate, and investigated laser oscillation and light-matter interactions in semiconductor quantum dot-nanocavity coupled systems. He was promoted to an Associate Professor in 2010 and has been investigating thermal conductivity nanoengineering and its application to thermoelectrics. 


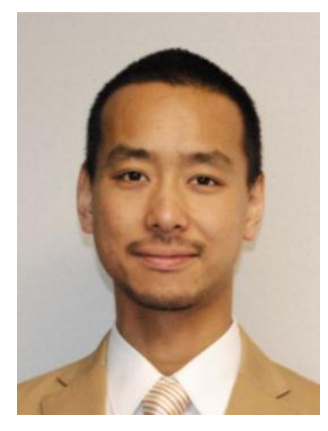

Junichiro Shiomi received the B.E. degree (1999) from Tohoku University, and Ph.D. degree (2004) from Royal Institute of Technology (KTH), Sweden. He is currently an Associate Professor in Department of Mechanical Engineering, The University of Tokyo. He is also a PRESTO researcher, Japan Science and Technology Agency. His research interests include heat conduction of nanomaterials, polymer composites, and thermoelectrics, phase change and fluidics in nanoscale, interfacial thermofluid dynamics, and thermal convections. He is a recipient of the Zeldovich Medal from the Committee on Space Research, and Young Scientists' Prize, the Commendation for Science and Technology by the Minister of Educational, Culture, Sports, Science and Technology. 


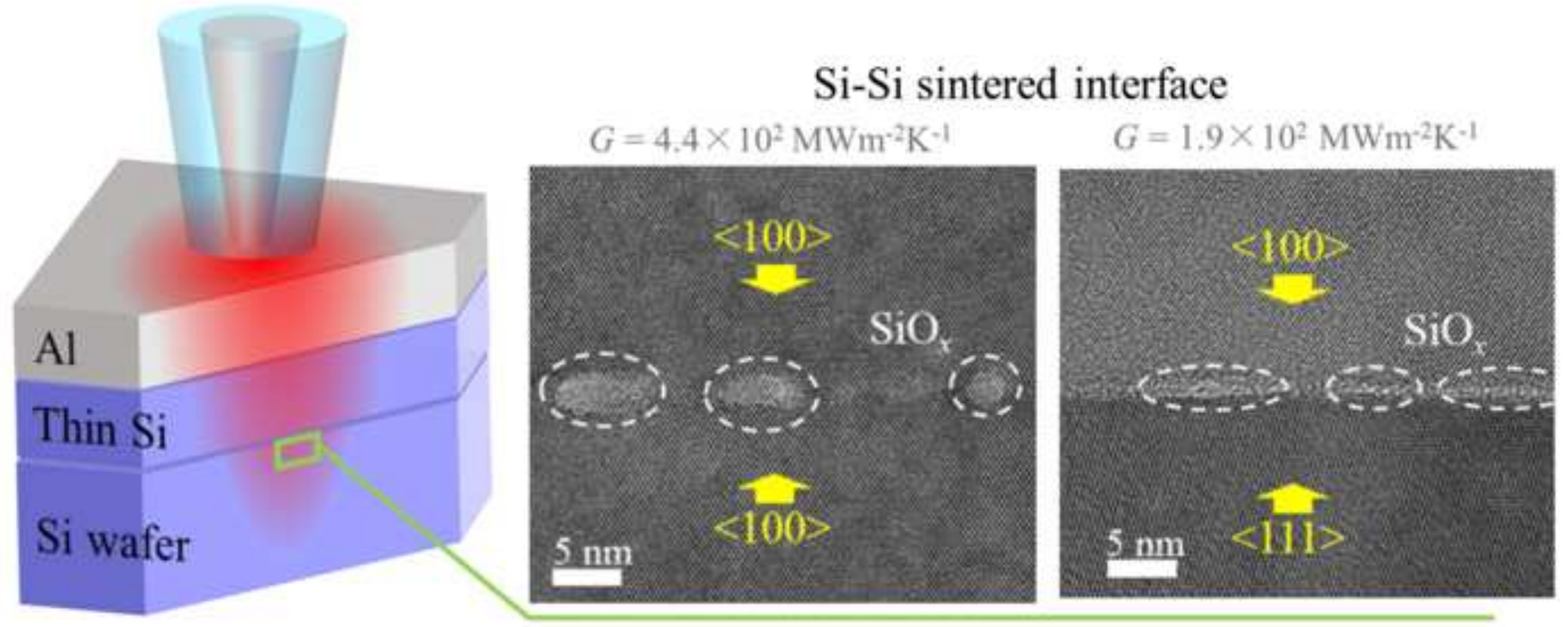

